Pteridines

Vol. 7, 1996, pp. 29-34

\title{
Neopterin Production, Degradation of Tryptophan and Neurologic Impairment in Human Immunodeficiency Virus Infection
}

\author{
Antonio Diez-Ruiz ${ }^{1 *}$, Arnulf A. Möller ${ }^{2}$, Gabriele Baier-Bitterlich ${ }^{1}$, Helmut Wachter ${ }^{1}$, and Dietmar \\ Fuchs $^{1 \S}$ \\ ${ }^{1}$ Institute of Medical Chemistry and Biochemistry, University of Innsbruck, and Ludwig Boltzmann In- \\ stitute of AIDS-Research Innsbruck, Austria \\ ${ }^{2}$ Max Planck Institute of Psychiatry, Clinical Institute, Munich, Germany
}

(Received March 9, 1996)

\begin{abstract}
Neurologic impairment in human immunodeficiency virus (HIV) infection may be related to metabolic factors in addition to a direct effect of HIV in the brain. An immune-enhanced tryptophan catabolism has been described in HIV infected patients that may contribute to neurologic/psychiatric symptoms. In $41 \mathrm{HIV}$-infected patients we studied the serum and cerebrospinal fluid concentrations of interferon- $\gamma$, neopterin, tryptophan and kynurenine in comparison to determinations of neurologic and psychiatric abnormalities as measured by a mental test and computerized tomography. Serum con centrations of interferon- $\gamma$, neopterin, and kynurenine were increased, whereas tryptophan levels were decreased in patients. Similarly, cerebrospinal fluid concentrations of neopterin and kynurenine were increased in the majority of patients compared to normal values. Significant relations existed between serum neopterin, tryptophan and its degradation product kynurenine and neurologic parameters. In cerebrospinal fluid, concentrations of neopterin but not of the other parameters tested were related to the mental state of patients. Our study demonstrates that systemic immune activation and its influence on tryptophan metabolism are related to neurologic disease in HIV patients. Intrathecal alterations of tryptophan and kynurenine appear to be less important.
\end{abstract}

Key words : Immune activation, HIV infection, Brain, Neopterin, Interferon- $\gamma$, Tryptophan

\section{Introduction}

Patients with human immunodeficiency virus (HIV) infection show a progressive loss of im-

$\S$ Address correspondence and reprit requests to: Dr. Dietmar Fuchs, Institute of Medical Chemistry and Biochemistry University of Innsbruck, Fritz Pregl Straße 3, A-6020 Innsbruck, Austria.

${ }^{*}$ Dr. Diez-Ruiz, University Hospital of Granada, is supported by "Fondo de Investigaciones Sanitarias", Ministry of Health, Spain. mune function that is associated with multiple findings of chronic immune activation (1-3), e.g increased ncopterin and interferon- $\gamma(\mathrm{IFN}-\gamma) \mathrm{con}$ centrations in body fluids have been described :? HIV infection $(3,4)$.

Neurologic impairment often develops in AIDS that is related to opportunistic infection a $a$ direct HIV neuronal infection leading $4 \mathrm{H:S}$ cephalitis (5). Nevertheless, because of $\therefore$ :sence of a clear cythopatic effect $\therefore \cdots: \cdots$ neuronal and glial cells, the possine

Pteridines $/$ Vol. $7 /$ No. 1 


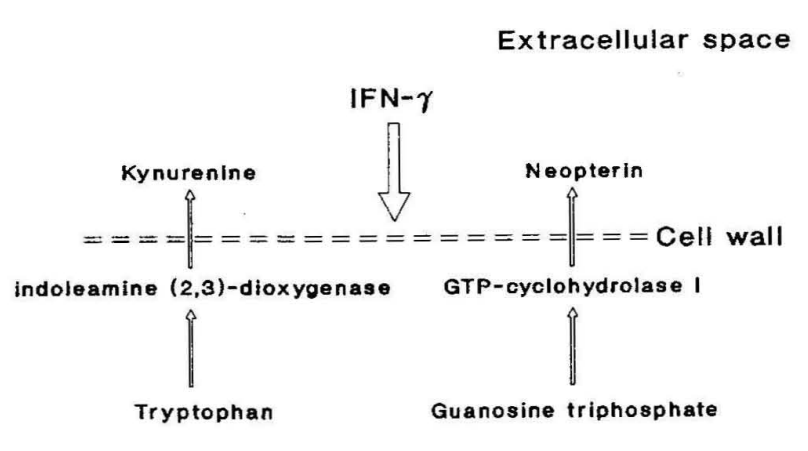

Macrophage + other cells

\section{Macrophage}

Figure 1. Scheme of the association between neopterin production and tryptophan degration induced by interferon- $\gamma$. The cytokine induces GTP-cyclohydrolase I and consequently neopterin production in human monocytes/macrophages; in parallel indoleamine $(2,3)$ dioxygenase is activated which degrades tryptophan via the $\mathrm{N}$-formyl-kynurenine pathway to produce kynurenine as a major component.

that an indirect effect of HIV may mediate the neurologic damage by activated cells of the brain like microglia/macrophages and by the synthesis of neurotoxic factors (6).

A role for the disturbed tryptophan metabolism in the pathogenesis of neurologic symptoms has been proposed (7 9) which is related to the chronic stimulation of macrophages and cytokine production. IFN- $\gamma$ activates indoleamine 2,3-dioxygenase (IDO), which causes a degradation of tryptophan forming kynurenine (Figure. 1) and other metabolites $(10,11)$. The calculation of a kynurenine to tryptophan ratio allows an estimate of IDO activity, it represents the quotient of the first product and substrate of the enzyme (8). The reduced bioavailability of tryptophan for the biosynthesis of serotonin and the accumulation of toxic compounds, such as kynurenic acid and quinolinic acid, as consequence of an enhanced induction of tryptophan catabolism by IFN- $\gamma$ may represent a relevant mechanism of the neurologic disturbances present in HIV infected patients (8).

In the present work we report on the relationship between serum and cerebrospinal fluid (CSF) concentrations of neoptcrin and IFN- $\gamma$, the tryptophan metabolism and indicators of brain damage in patients with different stages of HIV infection.

\section{Patients and Methods}

Forty-one patients (39 males, 2 females, age range: 21-54 years) in different stages of HIV-1 infection were investigated. The stage of infection was defined according to the Walter Reed Staging Classification (WR) system (12). Nine patients were in a WR stage below 5,15 were in WR 5 and 17 in WR 6.

All patients were neurologically examined and underwent cranial computerized tomography (CT). To test for neurologic destruction we quantified ventricular enlargement by measuring the ventricle-brain ratio in the CT. Cognitive functions were assessed by using the structured Interview for the Diagnosis of Alzheimer Dementia and Dementia of other Aetiology (SIDAM) (13). It is based on the diagnostic algorithm and criteria for diagnosing dementia according to ICD10 an DSM-III-R guidelines, it summarizes results of test performance in some different syndrome scales as orientation, memory, intellectual abilities (such as abstract thinking) and higher cortical functions (such as aphasia or apraxia). The maximal achievable score is $\mathbf{5 5}$.

Serum and/or CSF specimen were collected from patients. In total, sera of 32 and CSF of 23 patients were available for the study. There were 14 patients of whom matched serum and CSF specimens were available.

Serum and CSF neopterin concentrations were measured using radioimmunoassay (RIAcid, BRAHMS-Diagnostica, Berlin, Germany). The detection limit was $1 \mathrm{nmol} / 1$. Normal values (95th percentiles) are below $8.7 \mathrm{nmol} / \mathrm{l}$ in serum and below $6 \mathrm{nmol} / 1$ in CSF (14).

Tryptophan and kynurenine concentrations were measured by reverse-phase high pressure liquid chromatography as described (15). The normal serum values are $89.2 \pm 15.6 \mu \mathrm{mol} / 1$ for tryptophan and $2.1 \pm 0.49 \mu \mathrm{mol} / \mathrm{l}$ for kynurenine (15). The normal kynurenine to tryptophan ratio is $24.4 \pm 7.4$ (kynurenine in $\mu \mathrm{mol} / \mathrm{l}$ divided by tryptophan in $\mu \mathrm{mol} / 1$ multiplied with 1000). In CSF, normal values of tryptophan are higher than $1 \mu \mathrm{mol} / \mathrm{l}$ and of kynurenine below $0.1 \mu \mathrm{mol} / 1$ which is the detection limit of the method used.

IFN- $\gamma$ concentrations were quantified by radioimmunoassay (Centocor, Malvern, PA, USA). The sensitivity of the test was increased by employing an optimized assay procedure (3). The detection limit of the test is $18 \mathrm{U} / 1$ and the 95 th percentile in serum of healthy controls is $100 \mathrm{U} / \mathrm{l}$. Statistical evaluation of results was done by the non-parametric Wilcoxon rank test. For correlation analyses we used the Spearman rank correlation coefficients. 


\section{Results}

Seventeen of $32(53 \%)$ patients had increased serum levels of IFN- $\gamma, 28 / 32(87 \%)$ had increased neopterin and kynurenine serum concentrations as well and $24 / 32(75 \%)$ patients presented with decreased levels of tryptophan in serum (Table 1). When comparing the subgroups of patients according to the WR stage, scrum levels of neopterin were higher (mean \pm S.D.: $35.7 \pm$ 19.3 vs $27.9 \pm 16.9 \mathrm{nmol} / \mathrm{l}, \mathrm{p}=0.001)$ and serum tryptophan was lower $(43.7 \pm 12.4$ vs $54.3 \pm 16.6$ $\mu \mathrm{mol} / 1, \mathrm{p}<0.05)$ in patients with WR 6 stage than in patients with WR stage below 5 .

Serum levels of neopterin directly correlated to serum IFN- $\gamma(\mathrm{rs}=0.524, \mathrm{p}<0.005)$, serum kynurenine concentrations ( $\mathrm{rs}=0.407, \mathrm{p}<0.05)$ and to the serum kynurenine to tryptophan ratio ( $\mathrm{rs}=0.870$, $\mathrm{p}<0.0001)$. They were inversely correlated to serum tryptophan levels $(\mathrm{rs}=-0.692, \mathrm{p}<0.0001)$. In addition, serum IFN- $\gamma$ was related inversely to serum tryptophan $(\mathrm{rs}=-0.525, \mathrm{p}<0.01)$ and directly to the kynurenine to tryptophan ratio ( $\mathrm{rs}=0.555$, $\mathrm{p}<0.01$ ).

In CSF, 20 of 23 patients $(86 \%)$ had increased concentrations of neopterin, $17 / 23(73 \%)$ had increased kynurenine levels and 4 patients (17\%) showed decreased tryptophan levels. CSF concentrations of neopterin were related to concentrations of tryptophan ( $\mathrm{rs}=0.577, \mathrm{p}<0.01)$, kynurenine $(\mathrm{rs}=0.557, \mathrm{p}<0.01)$ and the CSF kynurenine to tryptophan ratio $(\mathrm{rs}=0.4 \mathrm{l} \mathrm{l}, \mathrm{p}<0.05)$. Tryptophan concentrations were related to kynu- renine levels ( $\mathrm{rs}=0.717, \mathrm{p}<0.00 \mathrm{I})$.

In examining correlations between serum and CSF parameters, serum neopterin was related to the CSF kynurenine to tryptophan ratio ( $\mathrm{rs}=0.533$, $\mathrm{p}<0.05)$. No other significant correlations were found across the blood/brain barrier.

The relations between neuropsychiatric abnormalities and the serum values of IFN- $\gamma$, neopterin, tryptophan and serum kynurenine to tryptophan ratio are shown in Table 2. Serum neopterin (Figure. 2) and the serum kynurenine to tryptophan ratio were inversely related to the SIDAM score reflecting cognitive impairment and directly to ventricle brain ratio in $\mathrm{CT}$, which monitors the size of brain lesions. Serum tryptophan was directly related to SIDAM (Figure. 2) and inversely to ventricle brain ratio. No significant correlations of serum kynurenine existed with SIDAM or ventricle brain ratio. In CSF, neopterin was inversely related to the SIDAM score $(\mathrm{rs}=-0.470, \mathrm{p}<0.05)$. No other relations were found between neurologic parameters and CSF determinations.

In the subgroup of 14 patients of whom both, serum and CSF, determinations were available, similar associations were found: serum neopterin was significantly related to SIDAM $(\mathrm{rs}=-0.568, \mathrm{p}$ $<0.05$ ) and to the ventricle brain ratio ( $\mathrm{rs}=0.613$, $\mathrm{p}<0.03)$. The association between serum tryptophan and neurologic evaluations did not reach statistical significance. CSF values of neopterin and tryptophan were not related to SIDAM or CT measurement.

Table 1. Values (mean \pm S.D.) of IFN- $\gamma$, neopterin, tryptophan and kynurenine in serum $(\mathrm{n}=32$ ) and CSF ( $\mathrm{n}=23$ ) of HIV infected patients.

\begin{tabular}{lcc}
\hline \hline & Serum & CSF \\
\hline IFN- $\gamma(\mathrm{U} / \mathbf{1})$ & $262 \pm 498$ & $150 \pm 231(\mathrm{U} / 1)$ \\
Neopterin $(\mathrm{nmol} / 1)$ & $23.9 \pm 16.1$ & $51.5 \pm 57.2(\mathrm{nmol} / 1)$ \\
Tryptophan $(\mu \mathrm{mol} / 1)$ & $57.3 \pm 17.9$ & $1.76 \pm 0.91(\mathrm{mmol} / 1)$ \\
Kynurenine $(\mu \mathrm{mol} / 1)$ & $3.47 \pm 0.94$ & $0.26 \pm 0.26$ \\
\hline
\end{tabular}

Table 2. Correlation coefficients and significance between serum parameters and cognitive impairement as reflected by the SIDAM test result and the ventricle/brain ratio by CT scan in 32 patients with HIV infection (n.s.=not significant).

\begin{tabular}{lll}
\hline \hline & SIDAM & CT \\
\hline IFN- $\gamma$ & $\mathrm{r}=-0.373$ & n.s. \\
Neopterin & $\mathrm{p}=0.041$ & $\mathrm{r}=0.579$ \\
Tryptophan & $\mathrm{r}=-0.658$ & $\mathrm{p}=0.004$ \\
& $\mathrm{p}=0.0004$ & $\mathrm{r}=-0.536$ \\
Kynurenine to & $\mathrm{r}=0.629$ & $\mathrm{p}=0.008$ \\
tryptophan ratio & $\mathrm{p}=0.0007$ & $\mathrm{r}=0.446$ \\
\hline
\end{tabular}



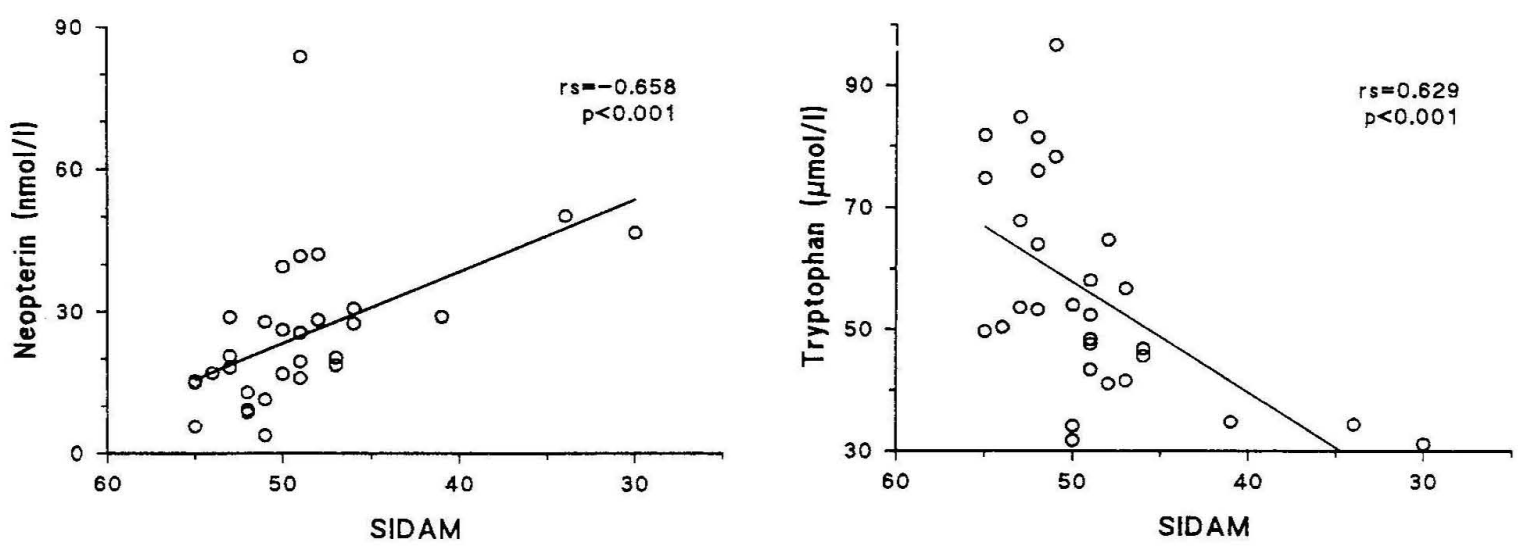

Figure 2. Correlations between SIDAM values (dementia score; lower levels indicating a higher degree of cognitive impairment) and serum concentrations of neopterin (left) and tryptophan (right); rs and $\mathrm{p}$ values stem from Spearman rank correlation analyses.

\section{Discussion}

Increased serum neopterin concentrations which were detected in the majority of our HIV patients indicate enhanced immune activation and concomittant endogenous formation of IFN- $\gamma$, because neopterin is released in large amounts by monocyte/macrophages upon stimulation with IFN- $\gamma$ (16). In fact, IFN- $\gamma$ concentrations were increased to a lesser frequency in our group of patients than neopterin, probably because IFN- $\gamma$ is rapidly cleared from plasma and circulating concentrations do not fully correspond to the actual amount of the cytokine released. In addition, other cytokines such as TNF- $\alpha$ are known to further enhance the effect of IFN- $\gamma$ on the macrophages regarding neopterin production in vitro. A close relationship between the IFN- $\gamma$, TNF- $\alpha$ and neopterin system was recently demonstrated also in vivo, when associations between soluble receptors for TNF- $\alpha$, neopterin and IFN- $\gamma$ have been shown in HIV infection and in other diseases $(17,18)$. These findings agree well with the chronic immune activation state present in HIV infection $(5,18,19)$.

Diminished tryptophan concentrations and increased kynurenine levels in serum argue in favour of an accelerated tryptophan catabolism in HIV patients. Kynurenine is a metabolite of the tryptophan degradation pathway. This metabolic process is catalyzed by the IDO and leads, in a first step, to the production of $\mathrm{N}$-formyl-kynurenine (Figure. 1), further products include quinolinic acid. In chronic immune activation states, such as congestive heart failure (20), Lyme neuroborreliosis (15), HIV infection (21) and mul- tiple sclerosis (22) an enhanced tryptophan catabolism has been described that is further supported by our study. Enhanced concentrations of kynurenine found in the patients with decreased tryptophan levels confirm that low tryptophan is not due to reduced dietary intake of the essential amino acid.

The correlations found in serum between IFN$\gamma$ and neopterin concentrations on the one hand, and the diminished tryptophan levels, increased kynurenine levels and increased kynurenine to tryptophan ratio on the other hand, confirm the relationship between chronic immune activation and enhanced tryptophan catabolism in HIV infection. In vitro, the induction of IDO and, consequently, the metabolism of tryptophan, is triggered by IFN- $\gamma$ (10). In vivo, the activation of IDO can be estimated by the kynurenine to tryptophan ratio. Therefore, as previously described, in HIV patients the tryptophan degradation is related to the chronic enhancement of immune activation in patients (23). Increased levels of neopterin and kynurenine and the correlations between neopterin and the products of tryptophan catabolism in the CSF provide further support for the conclusions drawn from serum data to be true also in the local compartment of the central nervous system.

The parameters used in our study to measure the neurologic and psychiatric impairment in patients with HIV infection were related to serum markers of immune activation and to indicators of enhanced tryptophan degradation. The mechanisms involved in the neurologic dysfunction of HIV infected patients may include a reduced availability of 5 -hydroxytryptamine (serotonin or 5- 
HT, Refs. 7, 8) or the accumulation of neurotoxins such as kynurenic acid and quinolinic acid $(8,11)$. Both circumstances would result from an exaggerated tryptophan catabolism. Therefore, the present study is consistent on the one hand with an involvement of the tryptophan metabolites in the HIV-associated neurologic symp toms and on the other hand with the responsibility of chronic immune stimulation in the development of this mechanism. The association found between the size of brain lesions, quantified by CT as ventricle/brain-ratios, and neopterin levels indicates that activated immunocompetent cells like macrophages and $\mathrm{T}$ lymphocytes could be involved in the destruction of cerebral tissue. However, a correlation not necessarily indicates a cause-effect relationship.

Interestingly, the presence of increased amounts of neopterin itself could have a role in the immunopathogenesis of brain lesions, because recently it has been shown that neopterin is capable of enhancing radical-mediated cytotoxic mechanisms (24). Thus, cellular cytotoxicity of immunocompetent cells within the brain might be enhanced in the presence of increased amounts of neopterin.

Noteworthy, the results in CSF are less conclusive. Only neopterin concentrations were related to the SIDAM score. It appears that the systemic chronic immune stimulation in HIV infection is more related to the tryptophan metabolism and the neurologic damage than local CNS phenomena. Although a protagonism has been proposed for activated brain macrophages in the secretion of neurotoxic products (6) our data point towards a more relevant role for systhemic metabolic disturbances on the origin of HIV-associated neurologic impairment which is probably in addition to local effects of HIV on brain tissue.

Unfortunately, the number of patients with matched serum and CSF specimens was rather low in this study. However, the evaluation of the 14 patients with matched material confirms the results obtained from the whole set of data, Certainly, a larger series of matched samples will be perferable for final conclusions.

\section{Acknowledgement}

This work was financially supported by the "Austrian funds "Zur Förderung der wissenschaftlichen Forschung", P10776".

\section{References}

1. Fauci AS. The human immunodeficiency virus: infectivity and mechanisms of pathogenesis. Science 1988; 239: 617.

2. Morrow WJW, Isemberg DA, Sobol RE, Stricker RB, Kieber Emmons T. AIDS virus infection and autoimmunity: a perspective of the clinical, immunological, and molecular origins of the autoallergic pathologies associated with HIV disease. Clin Immunol Immunopathol 1991; 58: 163-180.

3. Fuchs D, Hausen A, Reibnegger G, Werner ER, Werner-Felmayer G, Dierich MP, Wachter H. Interferon gamma concentrations are increased in sera from individuals infected with human immunodficiency virus type I. J Acquir Immune Defic Syndr 1989; 2: 158-162.

4. Fuchs D, Chiodi F, Albert J, AsjöB, Hagberg L, Hausen A, Norkrans G, Reibnegger G, Werner ER, Wachter $H$. Neopterin concentrations in cerebrospinal fluid of individuals infected with HIV-1. AIDS 1989; 3: 285-288.

5. Tyor WR, Glass JD, Baumrind N, McArthur JC; Griffin JW; BeckerPS, Griffin DE. Cytokine expression of macrophages in HIV-l-associated vacuolar myelopathy. Neurology 1993; 43: 1002-1009.

6. Achim CL, Heyes MP, Wiley CA. Quantitation of human immunodeficiency virus immune activation factors, and quinolinic acid in AIDS brains. J Clin In vest $1993 ; 91: 2769-2775$.

7. Larsson M, Hagberg L, Norkrans G, Forsman A. Indole amine deficiency in blood and cerebrospinal fluid from patients with human immunodeficiency virus infection. J Neurosci Res 1989; 23: 44l-446.

8. Fuchs D, Möller AA, Reibnegger G, Stöckle E, Werner ER, Wachter H. Decreased serum tryp tophan in patients with HIV-1 infection correlates with increased serum neopterin and with neurologic/ psychiatric symptoms. J Acquir Immune Defic Syndr 1990; 3: 873-876.

9. Heyes MP, Brew BJ, Martin A, Price RW, Salazar AM, Sidtis JJ, Yergey JA, Mouradian MM, Sadler AE, Keilp J, Rubinow D, Markey SP. Quinolinic acid in the cerebrospinal fluid an serum in HIV-1 infection: Relationship to clinical and neurological status. Ann Neurol 1991; 29: 202-209.

10. Werner-Felmayer G, Werner ER, Fuchs D, Hausen A, Reibnegger $G_{5}$-Wachter $H$. Neopterin formation and tryptophan degradation by a human myelomonocytic cell line (THP-1) upon cytokine treatment. Cancer Res 1990; 50: 2863-2867.

11. Heyes MP, Saito K, Markey SP. Human ma crophages convert L-tryptophan into the neurotoxin quinolinic acid. Biochem J 1992; 283: 633-635.

12. Redfield RR, Wright DC, Tramont EC. The WalterReed Staging classification for HTLV-III/LAV infection. New Engl J Med 1986; 314: 131-132.

13. Zaudig M, Mittelhammer J, Hiller W, Pauls A. Thora C, Moringo A, Mombour W. Description and test-retest reliability of the SIDAM-A structured $\ldots$ terview for the diagnosis of dementia of the A. zheimer type, multi-infarct dementia and Acme $=:$ of other etiology according to ICD-10 and $1 \mathrm{M}: \cdots$ 
R. Psychol Med 1991; $21: 225-236$.

14. Hagberg L, Dotevall L, Norkrans G, Larsson M, Wachter H, Fuchs D. Cerebrospinal fluid neopterin concentrations in central nervous system infection. J Infect Dis 1993; 168: 1285-1288.

15. Gasse T, Murr C, Meyersbach P, Schmutzhard E, Wachter H, Fuchs D. Neopterin production and tryptophan degradation in acuta Lyme neuroborreliosis versus late Lyme encephalopathy. Eur J Clin Chem Clin Biochem 1994; 32: 685-689.

16. Wachter H, Fuchs D, Hausen A, Reibnegger G, Werner ER. Neopterin as marker for activation of cellular immunity: Immunologic basis and clinical application. Adv Clin Chem 1989; 27: 81-141.

17. Zangerle R, Gallati H, Sarcletti M, Weiss G, Denz $\mathrm{H}$, Wachter $\mathrm{H}$, Fuchs $\mathrm{D}$. Increased serum concentrations of soluble tumor necrosis factor receptors in $\mathrm{HIV}$-infected individuals are associared with immune activation. J Acquir Immune Defic Syndr 1994; 7: 79-85.

18. Diez-Ruiz A, Tilz GP, Zangerle R, Baier-Bitterlich G, Wachter H, Fuchs D. Soluble receptors for tumor necrosis factor in clinical laboratory diagnosis. Eur J Haematol 1995; 54: 1-8.

19. Fuchs D, Hausen A, Reibnegger G, Werner ER, Dierich MP, Wachter H. Neopterin as a marker for activated cell-mediated immunity: Application in HIV infection. Immunol Today 1988; 9: 150-155.

20. Rudzite V, Skards JI, Fuchs D, Reibnegger G, Wachter $H$. Serum kynurenine and neopterin concentrations in patients with cardiomyopathy. Immunol Lett 1992; 32: 125-130.

21. Fuchs D, M"ller AA, Reibnegger G, Werner ER, Werner-Felmayer G, Dierich MP, Wachter H. Increased endogenous interferon-gamma and neopterin correlate with increased degradation of tryptophan in human immunodeficieny virus infection. Immunol Lett 1991; 28: 207-212.

22. Ott M, Demisch I, Engelhardt W, Fischer PA. Interleukin-2, soluble interleukin-2 receptor, neopterin, $\mathrm{L}$-tryptophan and $\beta 2$-microglobulin levels in CSF and serum of patients with relapsing-remitting or chronic progressive multiple sclerosis. J Neurol 1993; 241: 108-114.

23. Moeller AA, Fuchs D, Reibnegger G, Zaudig M, Werner ER, Wachter H. Changes of neopterin and tryptophan in serum and cerebrospinal fluid of patients in different stages of HIV-l infection. Pteridines $1990 ; 2: 91-93$.

24. Weiss G, Fuchs D, Hausen A, Reibnegger G, Werner ER, Werner-Felmayer G, Semenitz E, Dierich MP, Wachter $\mathrm{H}$. Neopterin modulates toxicity mediated by reactive oxygen and chloride species. FEBS Lett 1993; 1: 89-92. 